\title{
Optimal Parameter Updating for Optical Diffusion Imaging
}

\author{
J. C. Ye, K. J. Webb \\ Electrical and Computer Engineering \\ Purdue University \\ W. Lafayette, IN 47907
}

\author{
R. P. Millane, T. J. Downar \\ Computational Science and Engineering \\ Purdue University \\ W. Lafayette, IN 47907
}

\begin{abstract}
Because optical diffusion imaging is a highly nonlinear inverse problem, iterative inversion algorithms based on the Born approximation have usually been employed as reconstruction technique, but convergence is slow, especially for high contrast parameter distributions. We show here that the slow convergence of the conventional algorithms is due to the linear integral operator derived by the Born approximation not being the optimal Fréchet derivative. We derive the optimal Fréchet derivative operator with respect to the spatially varying absorption and scattering coefficients in integral form, and then develop a new iterative inversion algorithm.
\end{abstract}

\section{Introduction}

Optical diffusion imaging in highly scattering media such as tissue, as an alternative to x-ray tomography, presents significantly lower health risk, and also has successfully demonstrated its potential in biomedical applications. Since the optical inverse problem is nonlinear and ill-posed, iterative inversion algorithms for nonlinear inverse problems have been employed as solution techniques. However, current reconstruction algorithms for optical diffusion imaging have various properties that limit their convergence. This results from the approximation of integral form Fréchet derivative operator for the diffusion equation, and that two independent spatially varying parameters, the absorption and scattering coefficient, need to be reconstructed simultaneously. We will derive here the optimal Fréchet derivative operator with respect to the spatially varying absorption and scattering coefficients in integral form without any approximation. Numerical results are presented to illustrate the advantages of this approach.

\section{Previous Methods}

Consider a common form of the nonlinear ill-posed inverse problem :

$$
y=F(x) \quad, \quad\left\|y-y^{\delta}\right\|<\delta,
$$

where $F: X \rightarrow Y$ is a nonlinear operator between Hilbert spaces and $y^{\delta}$ is noisy data. Due to the illposedness of the nonlinear operator $F(\cdot)$, a regularized approximation to the solution is needed to make it depend continuously on the measurements $y^{\delta}$. The most famous approach is the Tikhonov regularization technique [1], which is formulated as

$$
\min _{x \in X} J_{\alpha}(x)=\left\|F(x)-y^{\delta}\right\|^{2}+\alpha\|x-\bar{x}\|^{2},
$$

where $\bar{x}$ is an initial estimate of the unknown parameter $x$ and $\alpha$ is the Lagrangian parameter. We can consider the following iterative technique for solution of (2):

$$
\begin{aligned}
x_{k+1}= & \bar{x}+\left(F^{\prime} \dagger\left(x_{k}\right) F^{\prime}\left(x_{k}\right)+\alpha I\right)^{-1} F^{\prime \dagger}\left(x_{k}\right) \\
& \times\left\{y^{\delta}-F\left(x_{k}\right)-F^{\prime}(x)\left[\bar{x}-x_{k}\right]\right\}
\end{aligned}
$$

which guarantees a solution that is a local minimum of (2). Note that in a conventional distorted Born iterative method (DBIM), or Newton-Kantorovich algorithm, $\bar{x}$ in (3) is successively replaced by previous estimates, $x_{k}[2]$.

Now consider the single-frequency diffusion equation that describes the optical modulation envelop[3, 4]

$$
\nabla \cdot(D \nabla \phi)+\left(-\mu_{a}+i \omega / c\right) \phi=-s \text { in } \Omega
$$

where $\Omega$ denotes a domain with boundary $\partial \Omega$, and $\left(\mu_{a}, \mu_{s}\right)$ denote the absorption and reduced scattering parameters, the diffusion coefficient $D$ is given by $\left[3\left(\mu_{a}+\mu_{s}\right)\right]^{-1}, c$ is the speed of light in the medium, and $\omega$ is the modulation angular frequency. Note that in this paper, $i$ denotes $\sqrt{-1}$. The flux, $\phi(\in Y)$, is generated by an excitation source $s$. Here, we have used the extrapolated zero-flux boundary condition on $\partial \Omega$ for simplicity [5].

Since we cannot find a nonlinear operator $F($.$) ex-$ plicitly from the PDE expression (4), it is quite difficult to apply the general form of the iterative inversion technique (3). However, in the conventional 
integral equation approach [3], $F(\cdot)$ is obtained using the approximation $\nabla \cdot(D \nabla \phi)$ as $D \nabla^{2} \phi$. With this approximation, (4) can be converted to

$$
\nabla^{2} \phi+k^{2} \phi=-\hat{s} \quad \text { in } \Omega, \phi=0 \text { on } \partial \Omega,
$$

where the unknown equivalent wavenumber-squared and modified source $\hat{s}$ are defined as

$k^{2}=3\left(\mu_{a}+\mu_{s}\right)\left(-\mu_{a}+i \omega / c\right), \hat{s}=3\left(\mu_{a}+\mu_{s}\right) s$

We can then identify the nonlinear operator $F(\cdot)$

$$
\begin{aligned}
F\left(k^{2}\right)= & \phi\left(r ; k^{b 2}\right) \\
& +\int_{\Omega} d r^{\prime} g\left(r, r^{\prime} ; k^{b 2}\right) \phi\left(r^{\prime} ; k^{b 2}\right) \Delta k^{2}\left(r^{\prime}\right)(7)
\end{aligned}
$$

and data $y=\phi\left(r ; k^{2}\right)$ where $k^{2}=k^{2}\left(\mu_{a}, \mu_{s}\right)$ is the unknown equivalent wave number-squared, and $k^{b 2}=$ $k^{2}\left(\mu_{a}^{b}, \mu_{s}^{b}\right)$ is the initial estimate. Note that $\Delta k^{2}\left(r^{\prime}\right)=$ $k^{2}\left(r^{\prime}\right)-k^{b 2}\left(r^{\prime}\right)$, and the Green's function, $g\left(r, r^{\prime} ; k^{b 2}\right)$ is that of the PDE in (5). Therefore, we can use the iteration (3) to obtain $\Delta k^{2}$ and convert it to the $\Delta \mu_{a}$ and $\Delta \mu_{s}$ as

$$
\begin{aligned}
\Delta \mu_{a} & =-\frac{\frac{\mu_{a}^{b}}{\omega / c} \operatorname{Im}\left[\Delta k^{2}\right]+\operatorname{Re}\left[\Delta k^{2}\right]}{\frac{\operatorname{Im}\left[\Delta k^{2}\right]}{\omega / c}+3\left(\mu_{a}^{b}+\mu_{s}^{b}\right)} \\
\Delta \mu_{s} & =\frac{\operatorname{Im}\left[\Delta k^{2}\right]}{3 \omega / c}-\Delta \mu_{a},
\end{aligned}
$$

where $\operatorname{Re}[\cdot]$ and $\operatorname{Im}[\cdot]$ denote the real and imaginary parts, respectively. This kind of procedure is known as the distorted Born iterative method (DBIM).

\section{The Optimal Fréchet Derivative in Integral Form}

The approach using (5) and (7) is not optimal for the following reasons. Firstly, the assumption that $\nabla \cdot(D \nabla \phi)=D \nabla^{2} \phi$ does not necessarily hold for large perturbations of $D$. Secondly, the integral equation is the Fréchet derivative operator for the unknown $k^{2}$, not for the unknown constitutive parameters, $\mu_{a}$ and $\mu_{s}$, which are required for reconstructing an image and relating results to material characteristics. Hence, the conventional approach provides a sub-optimal search direction, which results in slow convergence, especially when the perturbations in $\mu_{a}$ and $\mu_{s}$ are large.

This drawback can be overcome if we derive the Fréchet derivative $F^{\prime}(\cdot)$ directly from the diffusion equation (4) instead of the nonlinear functional $F(\cdot)$. Let $\left\{\psi_{k}\right\}_{k=1}^{K}$ be a partition of $\Omega$ such that $\Omega=$ $\cup_{k=1}^{K} \psi_{k}$. Suppose that $\mu_{a}$ and $\mu_{s}$ can be approximated as weighted sums of $\left\{\psi_{k}\right\}_{k=1}^{K}$, such that
$\mu_{a}=\sum_{k=1}^{K} \mu_{a}^{k} \psi_{k} \mu_{s}=\sum_{k=1}^{K} \mu_{s}^{k} \psi_{k}$. Then, the Fréchet derivative $F^{\prime}(\cdot)$ can be obtained as a generalized solution [6] of a new set of frequency-resolved diffusion equations.

We have shown [7] that for any $k$, the derivative of the generalized solution $u$ of (4) with respect to $\mu_{a}^{k}$ or $\mu_{s}^{k}$ converges uniformly to $v_{k}$ and $w_{k}$ in $H_{o}^{1}(\Omega)$, where $v_{k}$ and $w_{k}$ are generalized solutions of the following Dirichlet problems with zero flux boundary condition,

$$
L v_{k}=\frac{\nabla \cdot\left(\psi_{k} \nabla u\right)}{3\left(\mu_{a}^{k}+\mu_{s}^{k}\right)^{2}}+u \psi_{k} \quad L w_{k}=\frac{\nabla \cdot\left(\psi_{k} \nabla u\right)}{3\left(\mu_{a}^{k}+\mu_{s}^{k}\right)^{2}}
$$

where the frequency domain diffusion operator $L$ is defined as $L=\nabla \cdot D \nabla+\left(-\mu_{a}+\Im \omega / c\right)$. Moreover, if $\psi_{k} f=0, v_{k}$ and $w_{k}$ are generalized solutions of the following Dirichlet problem,

$$
L v_{k}=-\left(-1+\alpha^{k}\right) \psi_{k} u \quad L w_{k}=-\alpha^{k} \psi_{k} u
$$

where $\alpha^{k}=\left(-\mu_{a}^{k}+\Im \omega / c\right) /\left(\mu_{a}^{k}+\mu_{s}^{k}\right)$.

Using the above result and linearity, for $\Delta u_{a}=$ $\sum_{k=1}^{K} \Delta \mu_{a}^{k} \psi_{k}$ and $\Delta u_{s}=\sum_{k=1}^{K} \Delta \mu_{s}^{k} \psi_{k}$, we have

$$
\begin{aligned}
\phi\left(r ; \mu_{a}, \mu_{s}\right) & \simeq \phi\left(r ; \mu_{a}^{b}, \mu_{s}^{b}\right) \\
& +\sum_{k=1}^{K} g\left(r, r^{\prime} ; \mu_{a}^{b}, \mu_{s}^{b}\right)\left[-1+\alpha^{k}\right] \Delta \mu_{a}^{k} \psi_{k}\left(r^{\prime}\right) \\
& +\sum_{k=1}^{K} g\left(r, r^{\prime} ; \mu_{a}^{b}, \mu_{s}^{b}\right) \alpha^{k} \Delta \mu_{a}^{k} \psi_{k}\left(r^{\prime}\right)
\end{aligned}
$$

where the Green function $g(\cdot, \cdot)$ is that of (4). If $K \rightarrow$ $\infty$, it is not difficult to prove that (11) is finite and converges uniformly to the following integral equation:

$$
\begin{aligned}
\phi\left(r ; \mu_{a}, \mu_{s}\right) & =\phi\left(r ; \mu_{a}^{b}, \mu_{s}^{b}\right) \\
& +\int_{\Omega} d r^{\prime} g^{\prime}\left(r, r^{\prime} ; \mu_{a}^{b}, \mu_{s}^{b}\right) \phi\left(r^{\prime} ; \mu_{a}^{b}, \mu_{s}^{b}\right) O\left(r^{\prime}\right) \\
O\left(r^{\prime}\right) & =\left[-1+\alpha\left(r^{\prime}\right)\right] \Delta \mu_{a}\left(r^{\prime}\right)+\alpha\left(r^{\prime}\right) \Delta \mu_{s}\left(r^{\prime}\right)
\end{aligned}
$$

where $\alpha(r)=\left(-\mu_{a}^{b}(r)+\Im \omega / c\right) /\left(\mu_{a}^{b}(r)+\mu_{s}^{b}(r)\right)$.

\section{Parameter Transform}

Note the similarity between (7) and (12). However, the Green's function of (12) is derived from (4) without any approximation, while that of $(7)$ comes from the approximate PDE (5). The definition of $O\left(r^{\prime}\right)$ in (12) is also different from that of $\Delta k^{2}\left(r^{\prime}\right)$ in (7). Unlike the nonlinear rellationship between $\Delta k^{2}$ and $\left(\Delta \mu_{a}, \Delta \mu_{s}\right), O$ lies in the linear span of $\left(\Delta \mu_{a}, \Delta \mu_{s}\right)$. Therefore, we can guarantee that the proposed algorithm converges to a solution, and the convergence 
is faster than for the conventional DBIM approach. Since (12) and (7) are of identical form except for the definition of the unknown $\Delta k^{2}\left(r^{\prime}\right)$ and $O\left(r^{\prime}\right)$, we can use the same optimization techniques (3) as the conventional DBIM with the same order of complexity. The only difference is the conversion from $O$ to $\Delta \mu_{a}$ and $\Delta \mu_{s}$. From (12), the parameter transform can be explicitly represented using the previous estimate $\left(\mu_{a}^{b}, \mu_{s}^{b}\right)$ as

$$
\begin{aligned}
\Delta \mu_{a} & =-\frac{\mu_{a}^{b}}{\omega / c} \operatorname{Im}[O]-\operatorname{Re}[O] \\
\Delta \mu_{s} & =\frac{\mu_{a}^{b}+\mu_{s}^{b}}{w / c} \operatorname{Im}[O]-\Delta \mu_{a}
\end{aligned}
$$

\section{Simulation Results}

To examine the performance of our algorithm compared to the conventional DBIM, numerical simulations have been performed. All the simulations used "data" generated by solving (4). The optical modulation frequency used is $200 \mathrm{MHz}(=\omega / 2 \pi)$. A total of 12 sources and 12 detectors are located uniformly over the boundary of the $8 \times 8 \mathrm{~cm}^{2}$ domain, as shown in Fig. 1. The inhomogeneities are embedded in a homogeneous background medium with $\mu_{a}^{b}=0.02 \mathrm{~cm}^{-1}$ and $\mu_{s}^{b}=10.0 \mathrm{~cm}^{-1}$, and inhomogeneities in $\mu_{a}$ and $\mu_{s}$ are located at different positions with a $\mu_{a}$ peak of $0.12 \mathrm{~cm}^{-1}$ and a $\mu_{s}$ peak of $60 \mathrm{~cm}^{-1}$ at $(2 \mathrm{~cm}, 6 \mathrm{~cm})$ and $(6 \mathrm{~cm}, 2 \mathrm{~cm})$, respectively, as shown in Figs. 2(a)(b). The initial values for the estimate of $\mu_{a}$ and $\mu_{s}$ are $0.02 \mathrm{~cm}^{-1}$ and $10.0 \mathrm{~cm}^{-1}$, respectively. The reconstructed $\mu_{a}$ and $\mu_{s}^{\prime}$ for the conventional DBIM are shown in Figs. 3(a)-(b) after 6 iterations. After 6 iterations, no significant changes were not observed for both of the simulation results. In this case, the conventional DBIM failed to locate the inhomogeneities even qualitatively. However, use of the new algorithm described here, the quantitative value of the reconstruction is greatly improved, as shown in Figs. 3(c)(d). The elapsed time taken for 6 iterations by the new algorithm is 30 seconds on an Ultra Sparc workstation.

\section{Conclusion}

Even though the standard DBIM approach for the optical diffusion imaging problem has shown some success, the algorithm shows slow convergence especially when the spatial variation of $\mu_{a}$ and $\mu_{s}$ are large, since the Fréchet derivative they derived is not optimal for the unknown absorption and scattering parameters. In this paper, the optimal Fréchet derivative has been derived from the PDE and converted into a integral equation formulation. Numerical simulations show great improvement in the convergence properties of the new algorithm.

\section{References}

[1] A. Tikhonov and V. Arsein, Solution of Ill-posed Problem, V.H. Winston, Washington D.C., 1977.

[2] W. Chew, Waves and Fields in Inhomogeneous Media, Van Nostrand Reinhold, New York, 1990.

[3] Y. Yao, Y. Wang, Y. Pei, W. Zhu, and R. L. Barbour, "Frequency domain optical imaging of absorption and scattering distributions by a Born iterative method," J. Opt. Soc. Am. A 14, pp. 325342, Jan. 1997.

[4] H. Jiang, K. Paulsen, U. Osterberg, B. Pogue, and M. Patterson, "Optical image reconstruction using frequency-domain data: simulation and experiment," J. Opt. Soc. Am., A 13, pp. 253-266, Feb. 1996.

[5] J. J. Duderstadt and L. J. Hamilton, Nuclear Reactor Analysis, Wiley, New York, 1976.

[6] A. Friedman, Partial differential equations, Rinehart and Winston, Inc., New York:Holt, 1969.

[7] J. Ye, K. Webb, R. Millane, and T. Downar submitted to J. Opt. Soc. Am., A. . 


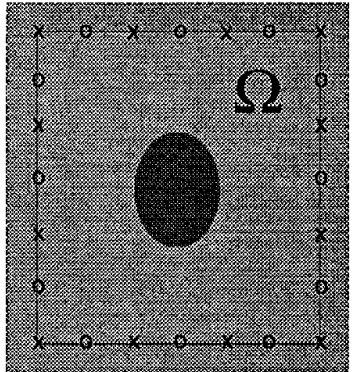

- Source

Location

$x$ Detector

Location

12 Sources

12 Detectors

Zero Flux Boundary

Figure 1: Data acquisition geometry for the full-angle profile inversion

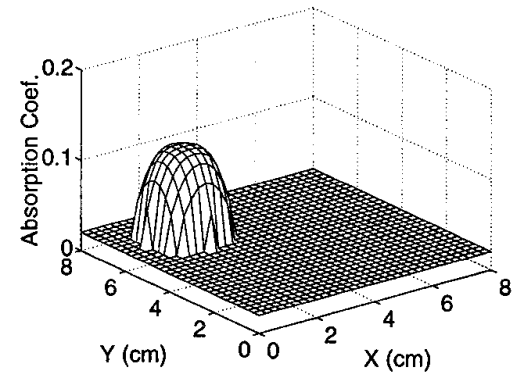

(a)

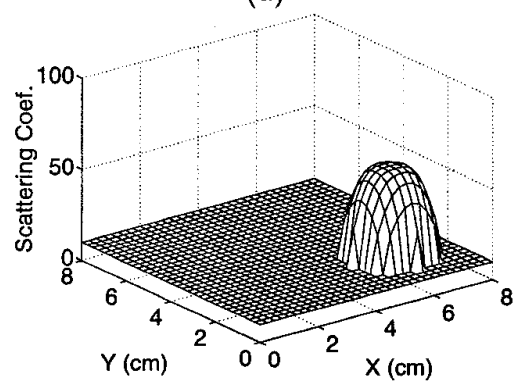

(b)

Figure 2: Original inhomogeneities in $\mu_{a}$ and $\mu_{s}$ in different locations. (a) Absorption and (b) scattering coefficient.

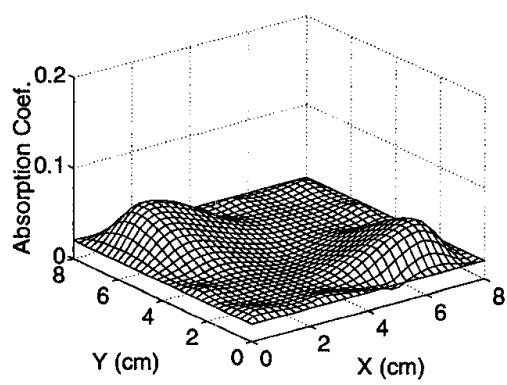

(a)

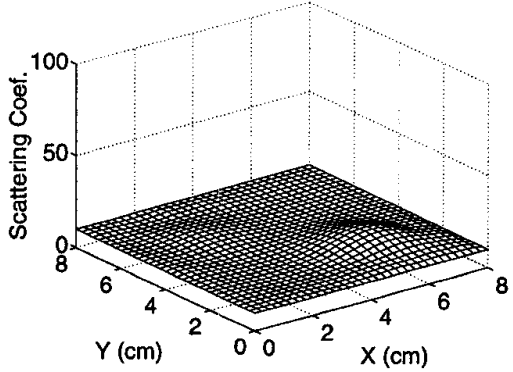

(b)

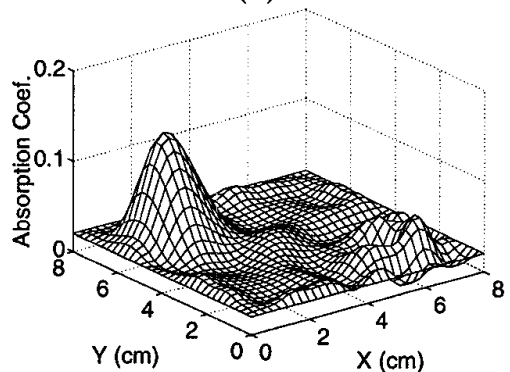

(c)

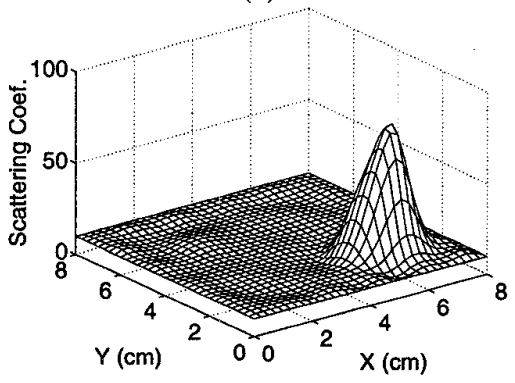

(d)

Figure 3: Reconstruction of inhomogeneities in $\mu_{a}$ and $\mu_{s}$ in different locations with $\mu_{a}$ peak of $0.12 \mathrm{~cm}^{-1}$ and $\mu_{s}$ peak of $60 \mathrm{~cm}^{-1}$. (a),(b) Reconstructed $\mu_{a}$ and $\mu_{s}$ by the conventional DBIM, and (c),(d) reconstructed $\mu_{a}$ and $\mu_{s}$ by the algorithm described here, after 6 iterations. 